\title{
On a conjecture related to the ruin probability for nonhomogeneous insurance claims
}

\author{
Raluca VERNIC
}

\begin{abstract}
Recently, nonhomogeneous claim sizes have been considered in the actuarial literature starting from the fact that the claims are seasonally influenced by the economic environment. In this context, Raducan et al. [8] obtained recursive formulas for the ruin probability at or before claim instants, and stated a conjecture that relates the order of the claims arrival to the magnitude of the corresponding ruin probability, conjecture supported by numerical examples. In this paper, we prove this conjecture in a particular case.
\end{abstract}

\section{Introduction}

One of the main tasks of actuaries consists of evaluating the ruin probability of insurance companies in order to establish their future financial politics (e.g., premiums calculations, reserves evaluation, reinsurance covers, surplus investments etc.). This implies studying the surplus (or risk) process of a company or of each of its business lines, which, at a certain time moment, is defined as the sum between the initial capital and the total premiums income, minus the sum of all the claims occurred by that time. The first time when this surplus becomes negative is the ruin moment. The ruin probability can be evaluated in finite time, over an infinite horizon (also called ultimate ruin probability; for a review of ruin probabilities, see, e.g., the book [1]), or at or

Key Words: continuous time surplus (risk) process, ruin probability, nonhomogeneous claim sizes, exponential distribution.

2010 Mathematics Subject Classification: 62P05, 91B30, 91B70.

Received: October, 2014.

Revised: January, 2015.

Accepted: February, 2015. 
before a certain claim instant. In this paper, we restrict to the last type of ruin probability, which is important because it shows where the risk process is relatively vulnerable to ruin (cf. [10], [11]). Therefore, we write the surplus process at the $n^{\text {th }}$ claim as

$$
U\left(T_{n}\right)=x-\sum_{i=1}^{n} \xi_{i},
$$

where $x=U(0)$ is the initial capital, $T_{n}$ is the moment when the $n^{\text {th }}$ claim occurs $\left(T_{0}=0\right)$, while $\xi_{n}=X_{n}-Y_{n}$ represents the loss between the $(n-1)^{t h}$ and the $n^{\text {th }}$ claim (included); here $Y_{n}$ is the firm's income between these claims and $X_{n}$ is the size of the $n^{t h}$ claim. Then the ruin probability at or before the $n^{\text {th }}$ claim instant is

$$
\psi_{n}(x)=P\left(\min _{1 \leq k \leq n} U\left(T_{k}\right)<0\right)=P\left(\max _{1 \leq k \leq n} \sum_{i=1}^{k} \xi_{i}>x\right) .
$$

The classical assumptions related to this risk process are: the claims occur according to a Poisson process, and their sizes are independent, identically distributed (i.i.d.) non-negative random variables (r.v.s). However, the homogeneity assumption related to the claims has been questioned after a tendency of increase of their sizes has been noticed due to several factors, like the economic environment, which cannot be neglected when studying the ruin over long time periods; for details on this phenomenon see [5] and the survey [6]. In this sense, several papers approached the finite time ruin probability for discrete-time risk processes with nonhomogeneous claim sizes, from which we mention [2], [3] and [4]. Recently, Raducan et al. [7] obtained recursive formulas for the ruin probability at or before claim instants, by considering two extensions of the Poisson claim number process and by taking the claim sizes to be independent, Erlang distributed, but non-i.d.. Further on, keeping the independent, non-i.d. Erlang claim sizes assumption, Raducan et al. [8] considered a claim number process even more general, assuming that the inter-claim incomes form a sequence of i.i.d. positive r.v.s following an arbitrary distribution. Moreover, related to the ruin probability evaluated for this last model at or before a certain claim instant, they stated a conjecture that relates the order of the claims arrival to the magnitude of the corresponding ruin probability, conjecture supported by numerical examples. Such a result provides upper and lower bounds for the estimated ruin probability for non-homogeneous claims. In this paper, we prove a particular case of this conjecture, which should be a starting point in proving the conjecture.

Therefore, in Section 2.1 we recall the formula of the ruin probability and the conjecture presented by [8], while in Section 2.2 we establish and prove a particular case. In the end, we present a few conclusions. 


\section{Main result}

In the following, we denote by $f^{(n)}$ the $n^{\text {th }}$ order derivative of a function $f$. By convention, $f^{(0)}=f$. We also denote by $\mathcal{L}_{Y}(a)$ the Laplace transform of the r.v. $Y$, i.e., $\mathcal{L}_{Y}(a)=E\left[e^{-a Y}\right]$.

Remark 2.1. An easy calculation shows that the Laplace transform $\mathcal{L}: \mathbb{R}_{+} \rightarrow$ $\mathbb{R}$ of a positive r.v. has the property that $\mathcal{L}^{(n)}(y)<0$ if $n$ is odd, while $\mathcal{L}^{(n)}(y)>0$ if $n$ is even, for any $n \in \mathbb{N}$ and $y>0$.

We also recall the definition of the stochastic dominance of two r.v.s, i.e., $X_{1} \prec s t X_{2} \stackrel{\text { def }}{\Leftrightarrow} F_{X_{1}} \geq F_{X_{2}}$, where $F_{X}$ is the cumulative distribution function of the r.v. $X$ (see, e.g., [9]). By convention, an empty product equals 1 and $0 !=1$.

\subsection{The conjecture}

Let us we denote by $\psi_{X_{n}, X_{n-1}, \ldots, X_{2}, X_{1} ; Y}$ the ruin probability at or before the $n^{\text {th }}$ claim when the claims arrive in the order $\left(X_{n}, X_{n-1}, \ldots, X_{2}, X_{1}\right)$ and the i.i.d. inter-claim incomes $\left(Y_{i}\right)_{i=1}^{n}$ are distributed as the generic r.v. $Y$. Related to this ruin probability, the following proposition has been proved in [8].

Proposition 2.2. Let $\left(X_{i}\right)_{i=1}^{n}$ be independent, exponentially distributed claims with different parameters, i.e., $X_{i} \sim \operatorname{Exp}\left(a_{i}\right)$, where $a_{i} \neq a_{j}, \forall i \neq j$, and let $\left(Y_{i}\right)_{i=1}^{n}$ be i.i.d. as a generic positive r.v. $Y$, and independent of the claims. Assuming that the order in which the claims arrive is $X_{n}, X_{n-1}, \ldots, X_{1}$, the ruin probability at or before the $n^{\text {th }}$ claim (which is the claim $X_{1}$ ) is given by

$$
\psi_{X_{n}, X_{n-1}, \ldots, X_{1} ; Y}(x)=\sum_{i=1}^{n} g_{n}\left(a_{i}\right) e^{-a_{i} x}, \forall x>0,
$$

where the coefficients $g_{n}$ are evaluated using the backward recursions

$$
\begin{aligned}
g_{1}\left(a_{1}\right) & =\mathcal{L}_{Y}\left(a_{1}\right) \\
g_{n+1}\left(a_{i}\right) & =g_{n}\left(a_{i}\right) \frac{a_{n+1}}{a_{n+1}-a_{i}} \mathcal{L}_{Y}\left(a_{i}\right), 1 \leq i \leq n, \\
g_{n+1}\left(a_{n+1}\right) & =\left(1+\sum_{i=1}^{n} g_{n}\left(a_{i}\right) \frac{a_{n+1}}{a_{i}-a_{n+1}}\right) \mathcal{L}_{Y}\left(a_{n+1}\right) .
\end{aligned}
$$

All the numerical examples presented in both [7] and [8] support the remark that the ruin probability corresponding to an arbitrary claims arrival order lies between the ruin probability corresponding to the decreasing stochastic order 
of the claims (which yields the largest ruin value) and the one corresponding to the increasing stochastic order of the claims (yielding the smallest ruin value). Therefore, it seems that the following conjecture holds:

Conjecture. If the claims are independent exponentially distributed, not necessarily $i$.d., and if $X_{1} \prec_{s t} X_{2} \prec_{s t} \ldots \prec_{s t} X_{n}$, then

$$
\psi_{X_{1}, X_{2}, \ldots, X_{n} ; Y}(x) \leq \psi_{X_{n}, X_{n-1}, \ldots, X_{1} ; Y}(x), \forall x>0 .
$$

This makes sense: according to [8], during the "small claims" period, the insurer's capital accumulates and, hence, it can face the "hard claims" period; however, if the larger claims come first, then the insurer's capital decreases and the ruin probability becomes larger. As proved in [8], this assertion is not true for any distribution of the claims.

\subsection{A particular case}

In the following, we shall prove a particular case of the above conjecture: more precisely, we assume that all the claims parameters are different and that $a_{1}>$ $a_{2}$ (which implies that $X_{1} \prec_{s t} X_{2}$ ), and show that $\psi_{X_{n}, \ldots, X_{3}, X_{2}, X_{1} ; Y}(x) \geq$ $\psi_{X_{n}, \ldots, X_{3}, X_{1}, X_{2} ; Y}(x), \forall x>0$. To do this, we need several lemmas.

Lemma 2.3. Let $f: \mathbb{R}_{+} \rightarrow \mathbb{R}$ be a function such that $f^{(n)}$ exists for all $n$ and $f^{(n)}(y)<0$ if $n$ is odd, while $f^{(n)}(y)>0$ if $n$ is even, for any $n$ and $y$. We define

$$
p_{a}(y ; f)=\left\{\begin{array}{c}
\frac{f(y)-f(a)}{a-y}, y \neq a \\
-f^{\prime}(a), y=a
\end{array} .\right.
$$

i) Then $p_{a}(y ; f)=p_{y}(a ; f)$.

ii) We have

$$
p_{a}^{(n)}(y ; f)=\left\{\begin{array}{l}
n !\left[\sum_{k=0}^{n} \frac{f^{(k)}(y)}{k !(a-y)^{n+1-k}}-\frac{f(a)}{(a-y)^{n+1}}\right], y \neq a \\
-\frac{f^{(n+1)}(a)}{n+1}, y=a
\end{array}, n \geq 0 .\right.
$$

iii) Moreover, $p_{a}^{(n)}(y ; f)$ has the same sign as $f^{(n)}(y)$. In particular, $p_{a}(y ; f)>$ 0 .

Proof. The equality in $(i)$ is obvious. We shall prove $(i i)$ by induction. If $n=0,(2.5)$ becomes

$$
p_{a}^{(0)}(y ; f)=\left\{\begin{array}{l}
\frac{f^{(0)}(y)}{a-y}-\frac{f(a)}{a-y}, y \neq a \\
-f^{\prime}(a), y=a
\end{array}=p_{a}(y ; f) .\right.
$$


We assume that (2.5) holds for $n$ and prove it for $n+1$. When $y \neq a$, we have

$$
\begin{gathered}
p_{a}^{(n+1)}(y ; f)=\left(p_{a}^{(n)}(y ; f)\right)^{\prime} \\
=n !\left[\sum_{k=0}^{n} \frac{1}{k !}\left(\frac{f^{(k+1)}(y)}{(a-y)^{n+1-k}}+\frac{(n+1-k) f^{(k)}(y)}{(a-y)^{n+2-k}}\right)-\frac{(n+1) f(a)}{(a-y)^{n+2}}\right] .
\end{gathered}
$$

Changing index $j=k+1$, the above sum becomes

$$
\begin{aligned}
& \sum_{k=0}^{n} \frac{1}{k !}\left[\frac{f^{(k+1)}(y)}{(a-y)^{n+1-k}}+\frac{(n+1-k) f^{(k)}(y)}{(a-y)^{n+2-k}}\right] \\
= & \sum_{j=1}^{n+1} \frac{1}{(j-1) !} \frac{f^{(j)}(y)}{(a-y)^{n+2-j}}+\sum_{k=0}^{n} \frac{(n+1-k) f^{(k)}(y)}{k !(a-y)^{n+2-k}} \\
= & \frac{(n+1) f^{(0)}(y)}{0 !(a-y)^{n+2-0}}+\sum_{k=1}^{n} \frac{(k+n+1-k) f^{(k)}(y)}{k !(a-y)^{n+2-k}} \\
& +\frac{n+1}{(n+1) !} \frac{f^{(n+1)}(y)}{(a-y)^{n+2-(n+1)}},
\end{aligned}
$$

which, inserted into (2.6) immediately yields (2.5) for $n+1$. To obtain the formula of $p_{a}^{(n+1)}(a ; f)$, we rewrite this result as

$$
\begin{gathered}
p_{a}^{(n+1)}(y ; f)=\frac{1}{a-y}\left[n !(n+1)\left(\sum_{k=0}^{n} \frac{f^{(k)}(y)}{k !(a-y)^{n+1-k}}-\frac{f(a)}{(a-y)^{n+1}}\right)\right. \\
\left.+f^{(n+1)}(y)\right]=\frac{1}{a-y}\left(f^{(n+1)}(y)+(n+1) p_{a}^{(n)}(y ; f)\right),
\end{gathered}
$$

where we take the limit for $y \rightarrow a$ and, using L'Hôspital's rule, we obtain

$$
\begin{aligned}
p_{a}^{(n+1)}(a ; f) & =\lim _{y \rightarrow a} \frac{f^{(n+2)}(y)+(n+1) p_{a}^{(n+1)}(y ; f)}{-1} \\
& =-\left[f^{(n+2)}(a)+(n+1) p_{a}^{(n+1)}(a ; f)\right]
\end{aligned}
$$

and thus $p_{a}^{(n+1)}(a ; f)=-f^{(n+2)}(a) /(n+2)$, which completes the proof of (ii).

To prove (iii), we use Taylor's expansion with Lagrange's remainder for $f(a)$, i.e.

$$
f(a)=\sum_{k=0}^{n} \frac{(a-y)^{k}}{k !} f^{(k)}(y)+\frac{(a-y)^{n+1}}{(n+1) !} f^{(n+1)}(\xi)
$$


where $\xi$ is between $a$ and $y$. We insert this formula into (2.5) for $y \neq a$ and obtain

$$
\begin{aligned}
p_{a}^{(n)}(y ; f)= & n !\left[\sum_{k=0}^{n} \frac{f^{(k)}(y)}{k !(a-y)^{n+1-k}}-\frac{1}{(a-y)^{n+1}}\right. \\
& \left.\times\left(\sum_{k=0}^{n} \frac{(a-y)^{k}}{k !} f^{(k)}(y)+\frac{(a-y)^{n+1}}{(n+1) !} f^{(n+1)}(\xi)\right)\right] \\
= & -\frac{n !}{(n+1) !} f^{(n+1)}(\xi),
\end{aligned}
$$

which, together with the formula of $p_{a}^{(n)}(a ; f)$, immediately yields the sign of $p_{a}^{(n)}(y ; f)$ for any $y$.

Let $\mathcal{L}$ be the Laplace transform of a r.v. and $f: \mathbb{R} \rightarrow \mathbb{R}$. For $a_{i}>0,1 \leq$ $i \leq n$ with $a_{i} \neq a_{j}, \forall i \neq j$, we recursively define the following functions

$$
\begin{aligned}
f_{2}\left(a_{1}, a_{2} ; f(\cdot)\right) & =\frac{\mathcal{L}\left(a_{2}\right) f\left(a_{2}\right)-\mathcal{L}\left(a_{1}\right) f\left(a_{1}\right)}{a_{1}-a_{2}} ; \\
f_{n}\left(a_{1}, \ldots, a_{n} ; f(\cdot)\right) & =f_{n-1}\left(a_{1}, \ldots, a_{n-1} ; f_{2}\left(a_{n}, \cdot ; f(\cdot)\right)\right), n \geq 3 .
\end{aligned}
$$

We also define

$$
h\left(a_{1}, a_{2}\right)=a_{1}\left(1-\mathcal{L}\left(a_{2}\right)\right)-a_{2}\left(1-\mathcal{L}\left(a_{1}\right)\right) .
$$

Lemma 2.4. With the above notation, it holds that

$$
f_{n}\left(a_{1}, \ldots, a_{n} ; f(\cdot)\right)=f_{2}\left(a_{1}, a_{2} ; f_{n-1}\left(a_{3}, \cdot, a_{4}, \ldots, a_{n} ; f(\cdot)\right)\right), n \geq 3 .
$$

Proof. We shall prove the result by induction. If $n=3$, formula $(2.10)$ is the same as (2.8). We assume that it holds for $n \geq 3$ and any $f$, and prove it for $n+1$. Successively using the definition (2.8), the induction hypothesis, the definition of $f_{2}$, then again the definition (2.8) and, finally, the definition of $f_{2}$, we obtain

$$
\begin{aligned}
& f_{n+1}\left(a_{1}, \ldots, a_{n+1} ; f(\cdot)\right)=f_{n}\left(a_{1}, \ldots, a_{n} ; f_{2}\left(a_{n+1}, \cdot ; f(\cdot)\right)\right) \\
& =f_{2}\left(a_{1}, a_{2} ; f_{n-1}\left(a_{3}, \cdot, a_{4}, \ldots, a_{n} ; f_{2}\left(a_{n+1}, \cdot ; f(\cdot)\right)\right)\right) \\
& =\frac{1}{a_{1}-a_{2}}\left[\mathcal{L}\left(a_{2}\right) f_{n-1}\left(a_{3}, a_{2}, a_{4}, \ldots, a_{n} ; f_{2}\left(a_{n+1}, \cdot ; f(\cdot)\right)\right)\right. \\
& \left.-\mathcal{L}\left(a_{1}\right) f_{n-1}\left(a_{3}, a_{1}, a_{4}, \ldots, a_{n} ; f_{2}\left(a_{n+1}, \cdot ; f(\cdot)\right)\right)\right] \\
& =\frac{1}{a_{1}-a_{2}}\left[\mathcal{L}\left(a_{2}\right) f_{n}\left(a_{3}, a_{2}, a_{4}, \ldots, a_{n}, a_{n+1} ; f(\cdot)\right)\right. \\
& \left.-\mathcal{L}\left(a_{1}\right) f_{n}\left(a_{3}, a_{1}, a_{4}, \ldots, a_{n}, a_{n+1} ; f(\cdot)\right)\right] \\
& =f_{2}\left(a_{1}, a_{2} ; f_{n}\left(a_{3}, \cdot, a_{4}, \ldots, a_{n}, a_{n+1} ; f(\cdot)\right)\right) \text {. }
\end{aligned}
$$


This completes the proof. $\square$

Lemma 2.5. Let $\mathcal{L}$ be the Laplace transform of a positive r.v. and let $f$ : $\mathbb{R}_{+} \rightarrow \mathbb{R}, f(y)=e^{-x y} \mathcal{L}(y)$ for some $x>0$. Then $f^{(n)}$ exists for all $n \in \mathbb{N}$, and $f^{(n)}(y)<0$ if $n$ is odd, while $f^{(n)}(y)>0$ if $n$ is even, for any $n$ and $y>0$.

Proof. We first prove by induction that

$$
f^{(n)}(y)=e^{-x y} \sum_{k=0}^{n}\left(\begin{array}{l}
n \\
k
\end{array}\right)(-x)^{n-k} \mathcal{L}^{(k)}(y) .
$$

When $n=0$, this gives $f^{(0)}(y)=e^{-x y} \mathcal{L}^{(0)}(y)=e^{-x y} \mathcal{L}(y)=f(y)$. We assume that the formula holds for $n \geq 0$ and prove it for $n+1$. We have

$$
\begin{aligned}
& f^{(n+1)}(y) \\
= & e^{-x y}\left[\sum_{k=0}^{n}\left(\begin{array}{l}
n \\
k
\end{array}\right)(-x)^{n-k} \mathcal{L}^{(k+1)}(y)-x\left(\sum_{k=0}^{n}\left(\begin{array}{l}
n \\
k
\end{array}\right)(-x)^{n-k} \mathcal{L}^{(k)}(y)\right)\right] \\
= & e^{-x y}\left[\mathcal{L}^{(n+1)}(y)+\sum_{k=1}^{n}\left(\left(\begin{array}{l}
n \\
k
\end{array}\right)+\left(\begin{array}{c}
n \\
k-1
\end{array}\right)\right)(-x)^{n+1-k} \mathcal{L}^{(k)}(y)\right. \\
& \left.-x^{n+1} \mathcal{L}(y)\right],
\end{aligned}
$$

where we changed the index $k+1$ in the first sum with $k$. Using now the formula $\left(\begin{array}{l}n \\ k\end{array}\right)+\left(\begin{array}{c}n \\ k-1\end{array}\right)=\left(\begin{array}{c}n+1 \\ k\end{array}\right)$, the stated formula of $f^{(n+1)}$ is immediate.

Based on Remark 2.1, we now note that the sign of $(-x)^{n-k} \mathcal{L}^{(k)}(y)$ is the same as the sign of $(-1)^{n}$ for any $k$, more precisely, $(-x)^{n-k} \mathcal{L}^{(k)}(y)<0$ if $n$ is odd and $(-x)^{n-k} \mathcal{L}^{(k)}(y)>0$ if $n$ is even, for any $k$. Then their sum after index $k$ ( $n$ being fixed) has the same sign as, e.g., $(-x)^{n} \mathcal{L}(y)$, and the proof is complete.

Lemma 2.6. If $\mathcal{L}$ is the Laplace transform of a positive r.v. and if $a_{1}>a_{2}>$ 0 , then

i) $h\left(a_{1}, a_{2}\right)>0$, where $h$ is defined in (2.9);

ii) Let $f_{n}$ be defined in (2.7)-(2.8), let $k(y)=e^{-x y}$ for some $x>0$, let $n \geq 2$ and $a_{i}>0$ with $a_{i} \neq a_{j}, \forall i \neq j, 1 \leq i \leq n$. Then,

$$
f_{n}\left(a_{1}, \ldots, a_{n} ; k(\cdot)\right)>0 .
$$

Proof. To prove $(i)$, we rewrite

$$
h\left(a_{1}, a_{2}\right)=a_{1} a_{2}\left(\frac{1-\mathcal{L}\left(a_{2}\right)}{a_{2}}-\frac{1-\mathcal{L}\left(a_{1}\right)}{a_{1}}\right)=a_{1} a_{2}\left(p_{0}\left(a_{2} ; \mathcal{L}\right)-p_{0}\left(a_{1} ; \mathcal{L}\right)\right)
$$


noting that $\mathcal{L}(0)=1$. Moreover, based on Remark 2.1, we can apply Lemma $2.3($ iii $)$, yielding $p_{0}^{\prime}(y ; \mathcal{L})<0$. It then follows that $p_{0}(y ; \mathcal{L})$ is a function strictly decreasing in $y$ and, since $a_{1}>a_{2}, p_{0}\left(a_{2} ; \mathcal{L}\right)>p_{0}\left(a_{1} ; \mathcal{L}\right)$, which completes the proof of $(i)$.

In order to obtain $(i i)$, we prove by induction after $n$ that

$$
\begin{aligned}
\frac{\partial^{m}}{\partial a_{2}^{m}} f_{n}\left(a_{1}, a_{2}, a_{3}, \ldots, a_{n} ; k(\cdot)\right) & <0 \text { if } m \text { is odd, } \\
\text { and } \frac{\partial^{m}}{\partial a_{2}^{m}} f_{n}\left(a_{1}, a_{2}, a_{3}, \ldots, a_{n} ; k(\cdot)\right) & >0 \text { if } m \text { is even; }
\end{aligned}
$$

if this result holds, then we immediately obtain $(i i)$ by taking $m=0$, which is even. We start with $n=2$, i.e., with

$$
f_{2}\left(a_{1}, a_{2} ; k(\cdot)\right)=\frac{e^{-a_{2} x} \mathcal{L}\left(a_{2}\right)-e^{-a_{1} x} \mathcal{L}\left(a_{1}\right)}{a_{1}-a_{2}}=p_{a_{1}}\left(a_{2} ; f(\cdot)\right),
$$

where $f(y)=e^{-x y} \mathcal{L}(y)$. We now apply Lemma 2.3 based on Lemma 2.5, and obtain the result that $p_{a_{1}}\left(a_{2} ; f(\cdot)\right)>0$. Assuming that the result holds for $n$, then, for $n+1$, we have from Lemma 2.4

$$
\begin{aligned}
f_{n+1}\left(a_{1}, \ldots, a_{n+1} ; k(\cdot)\right) & =f_{2}\left(a_{1}, a_{2} ; f_{n}\left(a_{3}, \cdot, a_{4}, \ldots, a_{n}, a_{n+1} ; k(\cdot)\right)\right) \\
& =p_{a_{1}}\left(a_{2} ; l(\cdot)\right),
\end{aligned}
$$

where $l(y)=\mathcal{L}(y) f_{n}\left(a_{3}, y, a_{4}, \ldots, a_{n}, a_{n+1} ; k(\cdot)\right)$. With a proof similar to the one of Lemma 2.5 , we easily obtain that

$$
l^{(m)}(y)=\sum_{j=0}^{m}\left(\begin{array}{c}
m \\
j
\end{array}\right) \mathcal{L}^{(j)}(y) \frac{\partial^{m-j}}{\partial y^{m-j}} f_{n}\left(a_{3}, y, a_{4}, \ldots, a_{n+1} ; k(\cdot)\right),
$$

to which we apply the induction hypothesis and the Remark 2.1 , and obtain that the sign of $l^{(m)}$ is the same as of $\mathcal{L}(y) \frac{\partial^{m}}{\partial y^{m}} f_{n}\left(a_{3}, y, a_{4}, \ldots, a_{n+1} ; k(\cdot)\right)$, i.e., the same sign of $\frac{\partial^{m}}{\partial y^{m}} f_{n}\left(a_{3}, y, a_{4}, \ldots, a_{n+1} ; k(\cdot)\right)$. Applying now Lemma $2.3(i i i)$ to $p_{a_{1}}\left(a_{2} ; l(\cdot)\right)$ yields the result for $n+1$, which completes the proof. $\square$

Lemma 2.7. Let $\left(X_{i}\right)_{i=1}^{n}$ be independent, exponentially distributed claims, $X_{i} \sim \operatorname{Exp}\left(a_{i}\right), a_{i} \neq a_{j}, \forall i \neq j$. As defined in formula (2.1), we denote by $g_{n}$ the coefficients of $\psi_{X_{n}, \ldots, X_{3}, X_{2}, X_{1} ; Y}$, and, similarly, by $g_{n}^{\prime}$ the coefficients of $\psi_{X_{n}, \ldots, X_{3}, X_{1}, X_{2} ; Y}$. Then, for a function $f$ and for each $n \geq 2$,

$D_{n}(f):=\sum_{i=1}^{n}\left(g_{n}\left(a_{i}\right)-g_{n}^{\prime}\left(a_{i}\right)\right) f\left(a_{i}\right)=\left(\prod_{i=3}^{n} a_{i}\right) h\left(a_{1}, a_{2}\right) f_{n}\left(a_{1}, \ldots, a_{n} ; f(\cdot)\right)$,

where $f_{n}$ and $h$ are defined in (2.7)-(2.9) for the positive r.v. Y. 
Proof. We prove the formula by induction. For $n=2$, we have from Proposition 2.2

$$
\begin{aligned}
& g_{1}\left(a_{1}\right)=\mathcal{L}_{Y}\left(a_{1}\right), g_{1}^{\prime}\left(a_{2}\right)=\mathcal{L}_{Y}\left(a_{2}\right), \\
& g_{2}\left(a_{1}\right)=\frac{a_{2}}{a_{2}-a_{1}} g_{1}\left(a_{1}\right) \mathcal{L}_{Y}\left(a_{1}\right)=\frac{a_{2}}{a_{2}-a_{1}} \mathcal{L}_{Y}^{2}\left(a_{1}\right), \\
& g_{2}^{\prime}\left(a_{2}\right)=\frac{a_{1}}{a_{1}-a_{2}} \mathcal{L}_{Y}^{2}\left(a_{2}\right), \\
& g_{2}\left(a_{2}\right)=\left(1+g_{1}\left(a_{1}\right) \frac{a_{2}}{a_{1}-a_{2}}\right) \mathcal{L}_{Y}\left(a_{2}\right), \\
& g_{2}^{\prime}\left(a_{1}\right)=\left(1+g_{1}^{\prime}\left(a_{2}\right) \frac{a_{1}}{a_{2}-a_{1}}\right) \mathcal{L}_{Y}\left(a_{1}\right),
\end{aligned}
$$

which yields

$$
\begin{aligned}
& D_{2}(f)= {\left[\frac{a_{2}}{a_{2}-a_{1}} \mathcal{L}_{Y}^{2}\left(a_{1}\right)-\left(1+\mathcal{L}_{Y}\left(a_{2}\right) \frac{a_{1}}{a_{2}-a_{1}}\right) \mathcal{L}_{Y}\left(a_{1}\right)\right] f\left(a_{1}\right) } \\
&+\left[\left(1+\mathcal{L}_{Y}\left(a_{1}\right) \frac{a_{2}}{a_{1}-a_{2}}\right) \mathcal{L}_{Y}\left(a_{2}\right)-\frac{a_{1}}{a_{1}-a_{2}} \mathcal{L}_{Y}^{2}\left(a_{2}\right)\right] f\left(a_{2}\right) \\
&= \mathcal{L}_{Y}\left(a_{1}\right) f\left(a_{1}\right)\left[\frac{a_{2} \mathcal{L}_{Y}\left(a_{1}\right)-a_{1} \mathcal{L}_{Y}\left(a_{2}\right)}{a_{2}-a_{1}}-1\right] \\
&+\mathcal{L}_{Y}\left(a_{2}\right) f\left(a_{2}\right)\left[1-\frac{a_{2} \mathcal{L}_{Y}\left(a_{1}\right)-a_{1} \mathcal{L}_{Y}\left(a_{2}\right)}{a_{2}-a_{1}}\right] \\
&=\quad\left[a_{1}\left(1-\mathcal{L}_{Y}\left(a_{2}\right)\right)-a_{2}\left(1-\mathcal{L}_{Y}\left(a_{1}\right)\right)\right] \frac{\mathcal{L}_{Y}\left(a_{1}\right) f\left(a_{1}\right)-\mathcal{L}_{Y}\left(a_{2}\right) f\left(a_{2}\right)}{a_{2}-a_{1}} \\
&=h\left(a_{1}, a_{2}\right) f_{2}\left(a_{1}, a_{2} ; f(\cdot)\right) .
\end{aligned}
$$

We assume that (2.11) holds for $n$ and any function $f$, and prove it for $n+1$. Using (2.3)-(2.4) from Proposition 2.2, we obtain

$$
g_{n+1}\left(a_{i}\right)-g_{n+1}^{\prime}\left(a_{i}\right)=\left\{\begin{array}{l}
\left(g_{n}\left(a_{i}\right)-g_{n}^{\prime}\left(a_{i}\right)\right) \frac{a_{n+1}}{a_{n+1}-a_{i}} \mathcal{L}_{Y}\left(a_{i}\right), 1 \leq i \leq n \\
\sum_{i=1}^{n}\left(g_{n}^{\prime}\left(a_{i}\right)-g_{n}\left(a_{i}\right)\right) \frac{a_{n+1}}{a_{n+1}-a_{i}} \mathcal{L}_{Y}\left(a_{n+1}\right), i=n+1
\end{array}\right.
$$

and hence, using the definition (2.7),

$$
D_{n+1}(f)=\sum_{i=1}^{n+1}\left(g_{n+1}\left(a_{i}\right)-g_{n+1}^{\prime}\left(a_{i}\right)\right) f\left(a_{i}\right)
$$




$$
\begin{aligned}
& =\sum_{i=1}^{n}\left(g_{n}\left(a_{i}\right)-g_{n}^{\prime}\left(a_{i}\right)\right) \frac{a_{n+1}}{a_{n+1}-a_{i}}\left[\mathcal{L}_{Y}\left(a_{i}\right) f\left(a_{i}\right)-\mathcal{L}_{Y}\left(a_{n+1}\right) f\left(a_{n+1}\right)\right] \\
& =a_{n+1} \sum_{i=1}^{n}\left(g_{n}\left(a_{i}\right)-g_{n}^{\prime}\left(a_{i}\right)\right) f_{2}\left(a_{n+1}, a_{i} ; f(\cdot)\right) .
\end{aligned}
$$

We now apply the induction hypothesis in which we replace the function $f(\cdot)$ with $f_{2}\left(a_{n+1}, \cdot ; f(\cdot)\right)$, and obtain

$$
D_{n+1}(f)=a_{n+1}\left(\prod_{i=3}^{n} a_{i}\right) h\left(a_{1}, a_{2}\right) f_{n}\left(a_{1}, \ldots, a_{n} ; f_{2}\left(a_{n+1}, \cdot ; f(\cdot)\right)\right),
$$

which, based on the definition (2.8), yields the result. This completes the proof.

Theorem 2.8. Under the assumptions of Lemma 2.7, if, moreover, $a_{1}>a_{2}>$ 0 (implying that $X_{1} \prec$ st $X_{2}$ ), it holds that for $x>0$,

$$
\left(\psi_{X_{n}, \ldots, X_{3}, X_{2}, X_{1} ; Y}-\psi_{X_{n}, \ldots, X_{3}, X_{1}, X_{2} ; Y}\right)(x)=\sum_{i=1}^{n}\left(g_{n}\left(a_{i}\right)-g_{n}^{\prime}\left(a_{i}\right)\right) e^{-a_{i} x}>0 .
$$

Proof. Clearly,

$$
\left(\psi_{X_{n}, \ldots, X_{3}, X_{2}, X_{1} ; Y}-\psi_{X_{n}, \ldots, X_{3}, X_{1}, X_{2} ; Y}\right)(x)=D_{n}(k),
$$

where $k(y)=e^{-x y}$. From (2.11), we have that

$$
\begin{aligned}
& \left(\psi_{X_{n}, \ldots, X_{3}, X_{2}, X_{1} ; Y}-\psi_{X_{n}, \ldots, X_{3}, X_{1}, X_{2} ; Y}\right)(x) \\
= & \left(\prod_{i=3}^{n} a_{i}\right) h\left(a_{1}, a_{2}\right) f_{n}\left(a_{1}, \ldots, a_{n} ; k(\cdot)\right) .
\end{aligned}
$$

Since $h$ and $f_{n}$ are defined based on the Laplace transform of the positive r.v. $Y$ and $a_{1}>a_{2}>0$, we can apply now both results in Lemma 2.6, and the proof is complete.

\section{Conclusions}

Note that, even if Theorem 2.8 presents a very particular case of the stated conjecture, its proof involves some mathematical effort. However, this particular case is a cornerstone for our future work, in which we intend to first extend the actual proof to the case

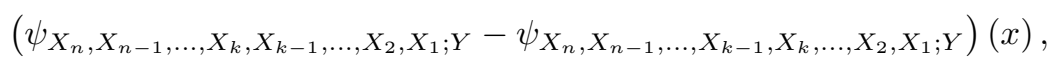


with all the exponential parameters of the claims different. Once this last case proved, we will be able to prove the conjecture under the same assumption of all parameters different, by noting that any permutation can be built by successively permuting two of its consecutive elements. Even more, while proving the conjecture in this way, we shall prove in fact the more general remark that the ruin probability corresponding to an arbitrary claims arrival order lies between the two extreme ruin probabilities given in the conjecture. Next step would be the proof of the general case when not all the exponential parameters are different, which is more cumbersome due to the complicate form of the recursions, see [8].

In practice, this result provides a starting point in obtaining upper and lower bounds for the estimated ruin probability when we note that the claims are exponentially distributed, but nonhomogeneous. On the same line, we also aim at obtaining some results for the ultimate ruin probability for noni.d. claims.

\section{Acknowledgement}

The author wishes to thank the two referees for their helpful suggestions that helped to revise the paper.

\section{References}

[1] Assmussen, S. and Albrecher, H., Ruin Probabilities, World Scientific Publishing Co., Singapore, 2010.

[2] Blazevicius, K.; Bieliauskiene, E. and Siaulys, J., Finite-time ruin probability in the inhomogeneous claim case, Lithuanian Mathematical Journal 50 (3), 2010, 260-270.

[3] Castaner, A.; Claramunt, M.M.; Gathy, M.; Lefevre, C. and Marmol, M., Ruin problems for a discrete time risk model with non-homogeneous conditions, Scandinavian Actuarial Journal (2), 2013, 83-102.

[4] De Kok, T.G., Ruin probabilities with compounding assets for discrete time finite horizon problems, independent period claim sized and general premium structure, Insurance: Mathematics and Economics 33, 2003, $645-658$.

[5] Lefevre, C. and Picard, P., A nonhomogeneous risk model for insurance, Computers and Mathematics with Applications 51, 2006, 325-334.

[6] Paulsen, J., Ruin models with investment income, Probability Surveys 5, 2008, 416-434. 
[7] Raducan, A.M., Vernic, R. and Zbaganu, G., Recursive calculation of ruin probabilities at or before claim instants for non-identically distributed claims, ASTIN Bulletin, vol. 45 (2), 2015, 421-443.

[8] Raducan, A.M., Vernic, R. and Zbaganu, G., On the ruin probability for nonhomogeneous claims and arbitrary inter-claim revenues, Journal of Computational and Applied Mathematics, 290, 2015, 319-333.

[9] Shaked, M. and Shanthikumar, J.G., Stochastic orders and their applications, Academic Press, New York, 1994.

[10] Stanford, D.A. and Stroinski, K.J., Recursive methods for computing finite-time ruin probabilities for phase-distributed claim sizes, ASTIN Bulletin 24 (2), 1994, 235-254.

[11] Stanford, D.A.; Stroinski, K.J. and Lee, K., Ruin probabilities based at claim instants for some non-Poisson claim processes, Insurance: Mathematics and Economics 26, 2000, 251-267.

Raluca VERNIC,

Faculty of Mathematics and Computer Science,

"Ovidius" University of Constanta,

124 Mamaia Blvd., 900527 Constanta, Romania

and Institute for Mathematical Statistics and Applied Mathematics,

Calea 13 Septembrie 13, 050711 Bucharest, Romania

Email: rvernic@univ-ovidius.ro 